\title{
An analysis of some functions that generalizes weighted means and OWA operators
}

\author{
Bonifacio Llamazares* \\ IMUVA (Institute of Mathematics) and Department of Applied Economics \\ University of Valladolid, 47011 Valladolid, Spain
}

\begin{abstract}
In this paper we analyze several classes of functions proposed in the literature to simultaneously generalize weighted means and OWA operators: WOWA operators, HWA operators and OWAWA operators. Since, in some cases, the results provided by these operators may be questionable, we introduce functions that also generalize both operators and characterize those satisfying a condition imposed to maintain the relationship among the weights.
\end{abstract}

\section{INTRODUCTION}

Weighted means and ordered weighted averaging (OWA) operators ${ }^{1}$ are wellknown functions widely used in the aggregation processes. Although both are defined through a weighting vector, their behavior is quite different: The weighted means allow to weight each information source in relation to their reliability while OWA operators allow to weight the values according to their ordering.

The need to combine both functions has been reported by several authors (see, among others, Torra ${ }^{2}$ and Torra and Narukawa ${ }^{3}$ ). For this reason, several classes of functions have appeared in the literature with the intent of simultaneously generalizing weighted means and OWA operators: the weighted OWA (WOWA) operator ${ }^{2}$, the hybrid weighted averaging (HWA) operator ${ }^{4}$ and the ordered weighted averaging-weighted average (OWAWA) operator ${ }^{5 ; 6}$. It is important to note that, in addition to the previous functions, there are others that have been used to combine both approaches (see, for instance, Yager ${ }^{7}$ and Engemann et al. ${ }^{8}$ ).

The aim of this paper is to analyze WOWA operators, HWA operators and OWAWA operators. Moreover, since in some cases the behavior and the results provided by these operators may be questionable, we propose to use functions

*e-mail: boni@eco.uva.es. 
that maintain the relationship between two initial weights of a weighting vector when the respective components of the other weighting vector are equal. In this way, we obtain a class of functions that have been previously introduced by Engemann et al. ${ }^{8}$ in a framework of decision making under risk and uncertainty.

The paper is organized as follows. In Section 2 we recall basic properties of functions and the definitions of weighted means, OWA operators and quantifiers. Section 3 is devoted to the analysis of the functions proposed in the literature to simultaneously generalize weighted means and OWA operators: WOWA operators, HWA operators and OWAWA operators. In Section 4 we propose a condition to maintain the relationship among the weights and characterize and analyze the functions that satisfy this condition. The paper concludes in Section 5 .

\section{PRELIMINARIES}

Throughout the paper we will use the following notation: $N=\{1, \ldots, n\} ; \# A$ will denote the cardinal of the set $A$; vectors will be denoted in bold; $\boldsymbol{\eta}$ will denote the vector $(1 / n, \ldots, 1 / n) ; \boldsymbol{x} \geq \boldsymbol{y}$ will mean $x_{i} \geq y_{i}$ for all $i \in\{1, \ldots, n\}$; given $\sigma$ a permutation of $\{1, \ldots, n\}, \boldsymbol{x}_{\sigma}$ will denote the vector $\left(x_{\sigma(1)}, \ldots, x_{\sigma(n)}\right)$.

In the following definition we present some well-known properties usually demanded to the functions used in the aggregation processes.

Definition 1. Let $F: \mathbb{R}^{n} \longrightarrow \mathbb{R}$ be a function.

1. $F$ is symmetric if for all $\boldsymbol{x} \in \mathbb{R}^{n}$ and for all permutation $\sigma$ of $N$ the following holds:

$$
F\left(\boldsymbol{x}_{\sigma}\right)=F(\boldsymbol{x})
$$

2. $F$ is monotonic if for all $\boldsymbol{x}, \boldsymbol{y} \in \mathbb{R}^{n}$ the following holds:

$$
\boldsymbol{x} \geq \boldsymbol{y} \Rightarrow F(\boldsymbol{x}) \geq F(\boldsymbol{y}) .
$$

3. $F$ is idempotent if for all $x \in \mathbb{R}$ the following holds:

$$
F(x, \ldots, x)=x .
$$

4. $F$ is compensative (also called internal) if for all $\boldsymbol{x} \in \mathbb{R}^{n}$ the following holds:

$$
\min (\boldsymbol{x}) \leq F(\boldsymbol{x}) \leq \max (\boldsymbol{x}) .
$$

5. $F$ is homogeneous of degree 1 if for all $\boldsymbol{x} \in \mathbb{R}^{n}$ and for all $\lambda>0$ the following holds:

$$
F(\lambda \boldsymbol{x})=\lambda F(\boldsymbol{x}) .
$$

Next we recall the definition of weighted means and OWA operators. It is worth noting that these functions are defined by means of vectors with nonnegative components whose sum is 1 . 
Definition 2. A vector $\boldsymbol{\mu} \in \mathbb{R}^{n}$ is a weighting vector if $\boldsymbol{\mu} \in[0,1]^{n}$ and $\sum_{i=1}^{n} \mu_{i}=$ 1 .

DeFINITION 3. Let $\boldsymbol{p}$ be a weighting vector. The weighted mean associated with $\boldsymbol{p}$ is the function $F_{\boldsymbol{p}}: \mathbb{R}^{n} \longrightarrow \mathbb{R}$ given by

$$
F_{\boldsymbol{p}}\left(x_{1}, \ldots, x_{n}\right)=\sum_{i=1}^{n} p_{i} x_{i} .
$$

Weighted means are monotonic, idempotent, compensative and homogeneous of degree 1 functions.

Yager $^{1}$ introduced OWA operators as a tool for aggregation procedures in multicriteria decision making. An OWA operator is similar to a weighted mean, but with the values of the variables previously ordered in a decreasing way. Thus, contrary to the weighted means, the weights are not associated with concrete variables. Consequently, OWA operators satisfy symmetry. Moreover, OWA operators also exhibit some other interesting properties such as monotonicity, idempotence, compensativeness and homogeneity of degree 1.

DEFINITION 4. Let $\boldsymbol{w}$ be a weighting vector. The OWA operator associated with $\boldsymbol{w}$ is the function $F^{\boldsymbol{w}}: \mathbb{R}^{n} \longrightarrow \mathbb{R}$ given by

$$
F^{\boldsymbol{w}}\left(x_{1}, \ldots, x_{n}\right)=\sum_{i=1}^{n} w_{i} x_{\sigma(i)},
$$

where $\sigma$ is a permutation of $N$ such that $x_{\sigma(1)} \geq \cdots \geq x_{\sigma(n)}$.

One of the most important issues in the theory of OWA operators is the determination of associated weights ${ }^{9 ; 10}$. OWA operators weights were related to quantifiers by Yager ${ }^{11}$.

Definition 5. A function $Q:[0,1] \longrightarrow[0,1]$ is a quantifier if it satisfies the following properties:

1. $Q(0)=0$.

2. $Q(1)=1$.

3. $x>y \Rightarrow Q(x) \geq Q(y)$; i.e., it is a non-decreasing function.

Given a quantifier $Q$, the OWA operator weights can be obtained from the following expression ${ }^{11}$ :

$$
w_{i}=Q\left(\frac{i}{n}\right)-Q\left(\frac{i-1}{n}\right), \quad i=1, \ldots, n .
$$

From this relation follows:

$$
Q\left(\frac{i}{n}\right)=\sum_{j=1}^{i} w_{j}, \quad i=1, \ldots, n
$$

i.e., the same weighting vector can be obtained through any quantifier interpolating the points $\left(i / n, \sum_{j=1}^{i} w_{j}\right), i=1, \ldots, n$. 


\section{Analysis of some generalizations of weighted means and OWA operators}

In this section we recall the definitions of some classes of functions proposed in the literature to simultaneously generalize weighted means and OWA operators: WOWA operators, HWA operators and OWAWA operators. Moreover, we illustrate with examples some questionable behaviors of these operators.

\subsection{WOWA operators}

WOWA operators ${ }^{2}$ were introduced by Torra in order to consider situations where both the importance of information sources and the importance of values had to be taken into account.

Definition 6. Let $\boldsymbol{p}$ and $\boldsymbol{w}$ be two weighting vectors. The WOWA operator associated with $\boldsymbol{p}$ and $\boldsymbol{w}$ is the function $W_{\boldsymbol{p}}^{\boldsymbol{w}}: \mathbb{R}^{n} \longrightarrow \mathbb{R}$ given by

$$
W_{\boldsymbol{p}}^{\boldsymbol{w}}\left(x_{1}, \ldots, x_{n}\right)=\sum_{i=1}^{n} \mu_{i} x_{\sigma(i)},
$$

where $\sigma$ is a permutation of $N$ such that $x_{\sigma(1)} \geq \cdots \geq x_{\sigma(n)}$ and the weight $\mu_{i}$ is defined as

$$
\mu_{i}=f\left(\sum_{j=1}^{i} p_{\sigma(j)}\right)-f\left(\sum_{j=1}^{i-1} p_{\sigma(j)}\right)
$$

where $f$ is a non-decreasing function that interpolates the points $\left(i / n, \sum_{j=1}^{i} w_{j}\right)$ together with the point $(0,0)$. Moreover, $f$ is the identity when the points can be interpolated in this way.

Different interpolation functions provide different results ${ }^{12}$. On the other hand, it is worth noting that any quantifier generating the weighting vector $\boldsymbol{w}$ satisfies the required properties of $f$ given in the previous definition (under the assumption that the quantifier is the identity when $\boldsymbol{w}=\boldsymbol{\eta}$ ). For this reason, it is possible to give an alternative definition of WOWA operators using quantifiers ${ }^{13}$.

DeFinition 7. Let $\boldsymbol{p}$ be a weighting vector and let $Q$ be a quantifier. The WOWA operator associated with $\boldsymbol{p}$ and $Q$ is the function $W_{\boldsymbol{p}}^{Q}: \mathbb{R}^{n} \longrightarrow \mathbb{R}$ given by

$$
W_{\boldsymbol{p}}^{Q}\left(x_{1}, \ldots, x_{n}\right)=\sum_{i=1}^{n} \mu_{i} x_{\sigma(i)},
$$

where $\sigma$ is a permutation of $N$ such that $x_{\sigma(1)} \geq \cdots \geq x_{\sigma(n)}$ and the weight $\mu_{i}$ is defined as

$$
\mu_{i}=Q\left(\sum_{j=1}^{i} p_{\sigma(j)}\right)-Q\left(\sum_{j=1}^{i-1} p_{\sigma(j)}\right)
$$


WOWA operators satisfy $W_{p}^{\eta}=F_{p}$ and $W_{\boldsymbol{\eta}}^{\boldsymbol{w}}=F^{\boldsymbol{w}}$. Moreover, they are monotonic, idempotent, compensative and homogeneous of degree 1 functions $^{2}$.

Although WOWA operators fulfills many interesting properties, they do not always provide the expected result as we show in the following example.

Example 1. Suppose we have four sensors to measure a certain physical property. The sensors are of different quality and precision, so they are weighted according to the weighting vector $\boldsymbol{p}=(0.5,0.2,0.2,0.1)$. Moreover, to prevent a faulty sensor alter the measurement, we take the weighting vector $\boldsymbol{w}=(0,0.5,0.5,0)$; thus, the maximum and minimum values are not considered.

Given $\boldsymbol{w}=(0,0.5,0.5,0)$, we have to choose a quantifier interpolating the points $(0,0),(0.25,0),(0.5,0.5),(0.75,1)$ and $(1,1)$. We consider the quantifier given by

$$
Q(x)= \begin{cases}0 & \text { if } x \leq 0.25 \\ 2 x-0.5 & \text { if } 0.25<x<0.75 \\ 1 & \text { if } x \geq 0.75\end{cases}
$$

which is depicted in Figure 1.

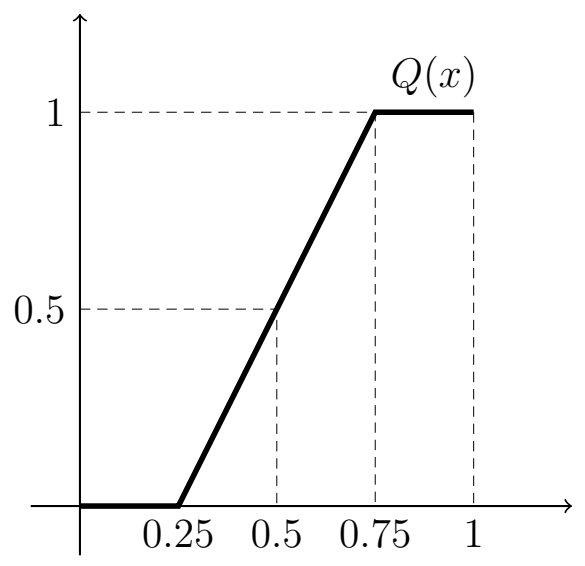

Figure 1: Quantifier associated to the weighting vector $\boldsymbol{w}=(0,0.5,0.5,0)$.

Suppose the values obtained by the sensors are $\boldsymbol{x}=(10,5,6,4)$. If $\sigma$ is a permutation ordering these values in a decrease way, then, in this case, $\boldsymbol{p}_{\sigma}=$ $\boldsymbol{p}=(0.5,0.2,0.2,0.1)$. The weighting vector $\boldsymbol{\mu}$ is

$$
\begin{aligned}
& \mu_{1}=Q(0.5)-Q(0)=0.5, \\
& \mu_{2}=Q(0.7)-Q(0.5)=0.4, \\
& \mu_{3}=Q(0.9)-Q(0.7)=0.1, \\
& \mu_{4}=Q(1)-Q(0.9)=0,
\end{aligned}
$$

and the value returned by the WOWA operator is

$$
W_{\boldsymbol{p}}^{\boldsymbol{w}}(10,5,6,4)=5+2.4+0.5=7.9 .
$$

However, our intention is not to consider the maximum and minimum values and only take into account the values 5 and 6 ; which have been provided by 
sensors with the same weight. Therefore, it seems logical to make the average of these values, in which case we would get 5.5 as final value.

It is worth noting that the WOWA operator returns a value greater than the two values that we want to aggregate ( 5 and 6 ) because it weights the maximum (10 in this case) with 0.5 . This weight is assigned by the WOWA operator to the first sensor when its value is the maximum or the minimum (regardless of the quantifier used). In fact, this is the minimum weight that the WOWA operator assigns to the first sensor. Therefore, the intended purpose of using the weighting vector $\boldsymbol{w}$ (not considering the maximum and minimum values) is not reached. Moreover, the weight assigned by the WOWA operator to the first sensor can be up to 1 ; for instance, when $\boldsymbol{p}=(0.5,0.25,0.2,0.05)$ and $\boldsymbol{x}=(9,10,5,4)$. In this case, only the value of the first sensor is taken into account. Note that the WOWA operator assigns a weight of zero to the value provided by the third sensor, $x_{3}=5$, even if this value is associated with the weights $p_{3}=0.2$ and $w_{3}=0.5$.

On the other hand, it is also important to emphasize that the weighting vectors $\boldsymbol{p}$ and $\boldsymbol{w}$ are not treated equally by the WOWA operator. For instance, when $p_{\sigma(i)}=0$, the value assigned by the WOWA operator to $x_{\sigma(i)}$ is

$$
\mu_{i}=Q\left(\sum_{j=1}^{i} p_{\sigma(j)}\right)-Q\left(\sum_{j=1}^{i-1} p_{\sigma(j)}\right)=0 .
$$

However, as we have seen above, this does not happen when $w_{i}=0$.

In addition to the previous shortcomings, there are other interesting properties that the WOWA operator does not satisfy (we continue assuming that $\boldsymbol{p}=(0.5,0.2,0.2,0.1)$ and $\boldsymbol{w}=(0,0.5,0.5,0))$ :

1. The value returned by the WOWA operator does not always lie between the values returned by the weighted mean and the OWA operator:

$$
\begin{aligned}
& F_{\boldsymbol{p}}(10,5,6,4)=5+1+1.2+0.4=7.6, \\
& F^{\boldsymbol{w}}(10,5,6,4)=3+2.5=5.5
\end{aligned}
$$

but $W_{\boldsymbol{p}}^{\boldsymbol{w}}(10,5,6,4)=7.9$.

2. The value returned by the WOWA operator does not always coincide with the values returned by the weighted mean and the OWA operator when both are equal:

$$
\begin{aligned}
F_{\boldsymbol{p}}(6.6,3,5,6) & =3.3+0.6+1+0.6=5.5, \\
F^{\boldsymbol{w}}(6.6,3,5,6) & =3+2.5=5.5
\end{aligned}
$$

but $W_{\boldsymbol{p}}^{\boldsymbol{w}}(6.6,3,5,6)=0.5 \cdot 6.6+0.2 \cdot 6+0.3 \cdot 5=6$. 


\subsection{HWA operators}

The second class of function that simultaneously generalize weighted means and OWA operators were introduced by $\mathrm{Xu}$ and $\mathrm{Da}^{4}$.

Definition 8. Let $\boldsymbol{p}$ and $\boldsymbol{w}$ be two weighting vectors. The HWA operator associated with $\boldsymbol{p}$ and $\boldsymbol{w}$ is the function $H_{\boldsymbol{p}}^{\boldsymbol{w}}: \mathbb{R}^{n} \longrightarrow \mathbb{R}$ given by

$$
H_{\boldsymbol{p}}^{\boldsymbol{w}}\left(x_{1}, \ldots, x_{n}\right)=\sum_{i=1}^{n} w_{i}\left(n p_{\sigma(i)} x_{\sigma(i)}\right),
$$

where $\sigma$ is a permutation of $N$ such that $p_{\sigma(1)} x_{\sigma(1)} \geq \cdots \geq p_{\sigma(n)} x_{\sigma(n)}$.

As we can see in the previous definition, the HWA operator associated with $\boldsymbol{p}$ and $\boldsymbol{w}$ is the composition of the OWA operator associated with $\boldsymbol{w}, F^{\boldsymbol{w}}$, with the function $H: \mathbb{R}^{n} \longrightarrow \mathbb{R}^{n}$ defined by

$$
H\left(x_{1}, \ldots, x_{n}\right)=\left(n p_{1} x_{1}, \ldots, n p_{n} x_{n}\right) .
$$

It is easy to check that $H_{p}^{\eta}=F_{p}$ and $H_{\eta}^{\boldsymbol{w}}=F^{\boldsymbol{w}}$ (see $\mathrm{Xu}$ and $\mathrm{Da}^{4}$ ). Moreover, it is also straightforward to check that HWA operators are monotonic and homogeneous of degree 1 functions. Nevertheless, they are neither idempotent nor compensative, as we show in the following example.

Example 2. Consider again the weighting vectors of Example 1, where $\boldsymbol{p}=$ $(0.5,0.2,0.2,0.1)$ and $\boldsymbol{w}=(0,0.5,0.5,0)$. If 10 is the value returned by all sensors, then the vector of components $n p_{i} x_{i}$ is $(20,8,8,4)$ and

$$
H_{p}^{\boldsymbol{w}}(10,10,10,10)=8 \neq 10 ;
$$

that is, $H_{\boldsymbol{p}}^{\boldsymbol{w}}$ is not idempotent. On the other hand, if the values obtained by the sensors are $\boldsymbol{x}=(10,5,6,8)$, then the vector of components $n p_{i} x_{i}$ is $(20,4,4.8,3.2)$, and

$$
H_{\boldsymbol{p}}^{\boldsymbol{w}}(10,5,6,8)=2.4+2=4.4<\min \{10,5,6,8\} ;
$$

i.e., $H_{p}^{\boldsymbol{w}}$ is not compensative.

\subsection{OWAWA operators}

Given that each value $x_{\sigma(i)}$ is associated with two weights, $p_{\sigma(i)}$ and $w_{i}$, we can consider a convex combination of these weights for obtaining a weight for $x_{\sigma(i)}$. This is the proposal made by Merigó ${ }^{5 ; 6}$.

Definition 9. Let $\boldsymbol{p}$ and $\boldsymbol{w}$ be two weighting vectors. The OWAWA operator associated with $\boldsymbol{p}$ and $\boldsymbol{w}$ is the function $O_{\boldsymbol{p}}^{\boldsymbol{w}}: \mathbb{R}^{n} \longrightarrow \mathbb{R}$ given by

$$
O_{\boldsymbol{p}}^{\boldsymbol{w}}\left(x_{1}, \ldots, x_{n}\right)=\sum_{i=1}^{n} v_{i} x_{\sigma(i)},
$$


where $\sigma$ is a permutation of $N$ such that $x_{\sigma(1)} \geq \cdots \geq x_{\sigma(n)}$ and the weight $v_{i}$ is defined as

$$
v_{i}=\alpha p_{\sigma(i)}+(1-\alpha) w_{i}
$$

with $\alpha \in[0,1]$.

Given that the OWAWA operator $O_{p}^{\boldsymbol{w}}$ can be written as a convex combination of $F_{p}$ and $F^{w}$,

$$
O_{\boldsymbol{p}}^{\boldsymbol{w}}\left(x_{1}, \ldots, x_{n}\right)=\alpha F_{\boldsymbol{p}}\left(x_{1}, \ldots, x_{n}\right)+(1-\alpha) F^{\boldsymbol{w}}\left(x_{1}, \ldots, x_{n}\right),
$$

it is straightforward to check that $O_{p}^{w}$ is monotonic, idempotent, compensative and homogeneous of degree 1. Moreover, the value returned by the OWAWA operator lies between the values returned by the weighted mean and the OWA operator, and coincides with them when both are equal.

However, it is not possible to find a fixed value of $\alpha$ for which $O_{\boldsymbol{p}}^{\eta}=F_{\boldsymbol{p}}$ and $O_{\boldsymbol{\eta}}^{\boldsymbol{w}}=F^{\boldsymbol{w}}$; that is, OWAWA operators do not generalize weighted means and OWA operators in this sense. Nevertheless, the previous property seems quite natural: if all the weights of a weighting vector are equal, then this weighting vector could be considered as a neutral vector in the aggregation process and only the other weighting vector should be taken into account.

In the following example we illustrate the behavior of OWAWA operators.

Example 3. Consider again the situation of Example 1, where $\boldsymbol{w}=(0,0.5,0.5,0)$, and suppose now we want to give the same importance to both weighting vectors $\boldsymbol{p}$ and $\boldsymbol{w}$. So, we take $\alpha=0.5$. Moreover, assume that the four sensors are equal; that is, $\boldsymbol{p}=\boldsymbol{\eta}=(0.25,0.25,0.25,0.25)$. Let's suppose that the first sensor is broken and that the values obtained by the sensors are $\boldsymbol{x}=(10,2,2,2)$. Then,

$$
O_{\boldsymbol{\eta}}^{\boldsymbol{w}}(10,2,2,2)=0.5 F_{\boldsymbol{\eta}}(10,2,2,2)+0.5 F^{\boldsymbol{w}}(10,2,2,2)=2+1=3,
$$

which is different from $F^{\boldsymbol{w}}(10,2,2,2)=2$.

\section{Choosing functions to maintain the relationship among the weights}

As we have seen in the previous section, OWAWA operators do not generalize weighted means and OWA operators in the sense that we want, while HWA operators are neither idempotent nor compensative. For their part, WOWA operators have good properties but they do not always return the expected value. Consider again Example 1, where $\boldsymbol{p}=(0.5,0.2,0.2,0.1)$ and $\boldsymbol{w}=(0,0.5,0.5,0)$, and take $\boldsymbol{x}=(10,5,4,6)$. Given that $\boldsymbol{w}=(0,0.5,0.5,0)$, we do not want to consider the maximum and minimum values and aggregate the remaining ones, in this case the values 5 and 6 . Since these values are given by sensors with weights 0.2 and 0.1 , respectively, one possibility is to weight these values by means of the weighting vector $(2 / 3,1 / 3)$; in this way, we maintain the relationship among the initial weights. 
According to the above remark, we look for a function $F_{\boldsymbol{p}}^{\boldsymbol{w}}: \mathbb{R}^{n} \longrightarrow \mathbb{R}$ given by

$$
F_{\boldsymbol{p}}^{\boldsymbol{w}}\left(x_{1}, \ldots, x_{n}\right)=\sum_{i=1}^{n} \rho_{i} x_{\sigma(i)},
$$

where $\sigma$ is a permutation of $N$ such that $x_{\sigma(1)} \geq \cdots \geq x_{\sigma(n)}$ and $\boldsymbol{\rho}$ is a weighting vector. Since each value $x_{\sigma(i)}$ is associated with the weights $p_{\sigma(i)}$ and $w_{i}$, we can consider a function $f:[0,1]^{2} \longrightarrow[0,1]$ that aggregates both weights, $f\left(w_{i}, p_{\sigma(i)}\right)$. From these values, we can obtain a weighting vector by defining $\rho_{i}$ as

$$
\rho_{i}=\frac{f\left(w_{i}, p_{\sigma(i)}\right)}{\sum_{j=1}^{n} f\left(w_{j}, p_{\sigma(j)}\right)} .
$$

Returning to the example above, if we want to maintain the relationship among the initial weights, the function $f$ has to satisfy the condition

$$
f(0.5,0.2)=2 f(0.5,0.1) .
$$

In the general case, it is necessary that $f$ satisfies the following condition:

$$
f(t x, y)=f(x, t y)=t f(x, y),
$$

for all $x, y \in[0,1]$ and $t \in[0, \infty)$ with $t x, t y \in[0,1]^{1}$. In the next proposition we characterize the functions that satisfy this condition.

Proposition 1. Let $f:[0,1]^{2} \longrightarrow[0,1]$ be a function such that

$$
f(t x, y)=f(x, t y)=t f(x, y)
$$

for all $x, y \in[0,1]$ and $t \in[0, \infty)$ with $t x, t y \in[0,1]$. Then $f(x, y)=$ cxy, where $c \in[0,1]$.

Proof. Given $x, y \in[0,1]$,

$$
f(x, y)=f(x \cdot 1, y \cdot 1)=x f(1, y \cdot 1)=x y f(1,1) .
$$

If $f(x, y)=c x y$, with $c \in[0,1]$, then

$$
\rho_{i}=\frac{w_{i} p_{\sigma(i)}}{\sum_{j=1}^{n} w_{j} p_{\sigma(j)}} ;
$$

that is,

$$
F_{\boldsymbol{p}}^{\boldsymbol{w}}\left(x_{1}, \ldots, x_{n}\right)=\frac{\sum_{i=1}^{n} w_{i} p_{\sigma(i)} x_{\sigma(i)}}{\sum_{j=1}^{n} w_{j} p_{\sigma(j)}} .
$$

\footnotetext{
${ }^{1}$ Note that the binary functions that satisfy $f(t x, y)=f(x, t y)$ for all $x, y \in[0,1]$ and $t \in[0, \infty)$ with $t x, t y \in[0,1]$ are called migratives ${ }^{14-17}$.
} 
It is worth noting that this function has been used by Engemann et al. ${ }^{8}$ in a framework of decision making under risk and uncertainty (in this case, $\boldsymbol{p}$ is the vector of probabilities of the states of nature).

In order to ensure that $F_{\boldsymbol{p}}^{\boldsymbol{w}}$ is well defined, we need that $w_{j} p_{\sigma(j)}$ be non-zero for some $j \in N$. This requirement is guaranteed by any of the following conditions:

1. The number of non-zero weights in each vector $\boldsymbol{p}$ and $\boldsymbol{w}$ is greater than $n / 2$.

2. All the components of $\boldsymbol{p}$ are non-zero.

It is important to point out that the last condition can be assumed without loss of generality (if any component of $\boldsymbol{p}$ is zero, this means that the weight of this information source is null; so it is not necessary to take it into account).

In addition to this, $F_{\boldsymbol{p}}^{\boldsymbol{w}}$ has another problem in your definition: sometimes, the vector $\boldsymbol{p}_{\sigma}$ is not unique and $F_{\boldsymbol{p}}^{\boldsymbol{w}}$ may return different values according to the vector $\boldsymbol{p}_{\sigma}$ used. This fact is illustrated in the following example.

Example 4. Consider again the weighting vectors $\boldsymbol{p}=(0.5,0.2,0.2,0.1)$ and $\boldsymbol{w}=$ $(0,0.5,0.5,0)$, and let $\boldsymbol{x}=(6,3,5,6)$. When $\boldsymbol{x}$ is ordered from greatest to least, then we have the vector $(6,6,5,3)$. In this vector, the first component can be associated to the weight $p_{1}=0.5$ or $p_{4}=0.1$. In the first case, the weighting vector $\boldsymbol{\rho}$ is $(0,1 / 3,2 / 3,0)$ and

$$
F_{\boldsymbol{p}}^{\boldsymbol{w}}(6,3,5,6)=2+\frac{10}{3}=\frac{16}{3}
$$

In the second case, the weighting vector $\boldsymbol{\rho}$ is $(0,5 / 7,2 / 7,0)$ and

$$
F_{\boldsymbol{p}}^{\boldsymbol{w}}(6,3,5,6)=\frac{30}{7}+\frac{10}{7}=\frac{40}{7} .
$$

This behavior is related to the following fact:

$$
\lim _{x \rightarrow 6^{+}} F_{\boldsymbol{p}}^{\boldsymbol{w}}(x, 3,5,6)=\frac{16}{3},
$$

but

$$
\lim _{x \rightarrow 6^{-}} F_{\boldsymbol{p}}^{\boldsymbol{w}}(x, 3,5,6)=\frac{40}{7} .
$$

A similar problem arises in the IOWA operators, introduced by Yager and Filev $^{18}$. The solution proposed by these authors, applied to our framework, is to replace the weights associated to equal values by the average of them. In the previous example we replace the weights $p_{1}=0.5$ and $p_{4}=0.1$ by 0.3 . In this case, the weighting vector $\boldsymbol{\rho}$ is $(0,3 / 5,2 / 5,0)$

$$
F_{\boldsymbol{p}}^{\boldsymbol{w}}(6,3,5,6)=\frac{18}{5}+2=\frac{28}{5}
$$


It is worth noting that the behavior of this function is similar to a weighted mean, but where the weighting vector varies depending on $\boldsymbol{x}^{2}$. For this reason, $F_{\boldsymbol{p}}^{\boldsymbol{w}}$ is idempotent and compensative. Moreover, it is easy to check that $F_{\boldsymbol{p}}^{\boldsymbol{w}}$ is homogeneous of degree 1 and that $F_{\boldsymbol{p}}^{\boldsymbol{\eta}}=F_{\boldsymbol{p}}$ (Engemann et al. ${ }^{8}$ ) and $F_{\boldsymbol{\eta}}^{\boldsymbol{w}}=F^{\boldsymbol{w}}$.

Nevertheless, as noted by $\mathrm{Liu}^{21}, F_{\boldsymbol{p}}^{\boldsymbol{w}}$ is not monotonic. In fact, as we show in the following example, $F_{\boldsymbol{p}}^{\boldsymbol{w}}$ is not monotonic although the non-zero components of the weighting vector $\boldsymbol{w}$ are equal.

Example 5. Consider again the weighting vectors $\boldsymbol{p}=(0.5,0.2,0.2,0.1)$ and $\boldsymbol{w}=$ $(0,0.5,0.5,0)$. Then, we have:

$$
\begin{aligned}
& F_{\boldsymbol{p}}^{\boldsymbol{w}}(5.8,5,6,4)=\frac{29}{7}+\frac{10}{7}=\frac{39}{7} \\
& F_{\boldsymbol{p}}^{\boldsymbol{w}}(10,5,6,4)=3+2.5=5.5
\end{aligned}
$$

However, in some cases, the functions $F_{p}^{\boldsymbol{w}}$ present a monotonic behavior; for instance, when the coordinates of both points can be ordered in a decreasing way with the same permutation (in order to avoid problems with their definition, we only consider points without repeated coordinates).

Proposition 2. Let $\boldsymbol{x}, \boldsymbol{y} \in \mathbb{R}^{n}$ such that $\#\left\{x_{i} \mid i \in N\right\}=\#\left\{y_{i} \mid i \in N\right\}=n$ and let $\sigma$ be a permutation of $N$ such that $x_{\sigma(1)}>\cdots>x_{\sigma(n)}$ and $y_{\sigma(1)}>\cdots>$ $y_{\sigma(n)}$. If $\boldsymbol{x} \geq \boldsymbol{y}$, then $F_{\boldsymbol{p}}^{\boldsymbol{w}}(\boldsymbol{x}) \geq F_{\boldsymbol{p}}^{\boldsymbol{w}}(\boldsymbol{y})$.

Proof. Given $\boldsymbol{x}, \boldsymbol{y} \in \mathbb{R}^{n}$ such that $\boldsymbol{x} \geq \boldsymbol{y}$ and $\sigma$ a permutation of $N$ such that $x_{\sigma(1)}>\cdots>x_{\sigma(n)}$ and $y_{\sigma(1)}>\cdots>y_{\sigma(n)}$, we have

$$
F_{\boldsymbol{p}}^{\boldsymbol{w}}\left(x_{1}, \ldots, x_{n}\right)=\frac{\sum_{i=1}^{n} w_{i} p_{\sigma(i)} x_{\sigma(i)}}{\sum_{j=1}^{n} w_{j} p_{\sigma(j)}} \geq \frac{\sum_{i=1}^{n} w_{i} p_{\sigma(i)} y_{\sigma(i)}}{\sum_{j=1}^{n} w_{j} p_{\sigma(j)}}=F_{\boldsymbol{p}}^{\boldsymbol{w}}\left(y_{1}, \ldots, y_{n}\right) .
$$

A specific case of the previous situation happens when only a information source changes its value. For instance, suppose that $\left(x_{1}^{0}, \ldots, x_{i}^{0}, \ldots, x_{n}^{0}\right)$ are the values provided by the information sources and $\sigma$ is a permutation of $N$ such that $x_{\sigma(1)}^{0}>\cdots>x_{\sigma(i)}^{0}>\cdots>x_{\sigma(n)}^{0}$. Given that the value $x_{i}^{0}$ occupies the $\sigma^{-1}(i)$ th position $\left(\right.$ since $\left.x_{\sigma\left(\sigma^{-1}(i)\right)}^{0}=x_{i}^{0}\right)$, we have

$$
x_{\sigma\left(\sigma^{-1}(i)-1\right)}^{0}>x_{i}^{0}>x_{\sigma\left(\sigma^{-1}(i)+1\right)}^{0} .
$$

Therefore, the function of one variable $F_{\boldsymbol{p}}^{\boldsymbol{w}}\left(x_{1}^{0}, \ldots, x_{i-1}^{0}, x, x_{i+1}^{0}, \ldots, x_{n}^{0}\right)$ is monotonic when $x \in\left(x_{\sigma\left(\sigma^{-1}(i)+1\right)}^{0}, x_{\sigma\left(\sigma^{-1}(i)-1\right)}^{0}\right)$.

On the other hand, similar to WOWA operators, there are other interesting properties that the function $F_{\boldsymbol{p}}^{\boldsymbol{w}}$ does not satisfy. If we consider the weighting vectors $\boldsymbol{p}=(0.5,0.2,0.2,0.1)$ and $\boldsymbol{w}=(0,0.5,0.5,0)$, then:

\footnotetext{
${ }^{2}$ This behavior is also seen in OWA operators and mixture operators. These last functions were introduced by Marques Pereira and Pasi ${ }^{19}$ and they are a particular case of Bajraktarević means ${ }^{20}$.
} 
1. The value returned by $F_{p}^{w}$ does not always lie between the values returned by the weighted mean and the OWA operator:

$$
\begin{aligned}
F_{\boldsymbol{p}}(10,3,5,6) & =5+0.6+1+0.6=7.2 \\
F^{\boldsymbol{w}}(10,3,5,6) & =3+2.5=5.5
\end{aligned}
$$

but $F_{\boldsymbol{p}}^{\boldsymbol{w}}(10,3,5,6)=16 / 3$

2. The value returned by $F_{\boldsymbol{p}}^{\boldsymbol{w}}$ does not always coincide with the value returned by the weighted mean and the OWA operator when both values are the same:

$$
\begin{aligned}
F_{p}(6.6,3,5,6) & =3.3+0.6+1+0.6=5.5, \\
F^{\boldsymbol{w}}(6.6,3,5,6) & =3+2.5=5.5,
\end{aligned}
$$

but $F_{p}^{\boldsymbol{w}}(6.6,3,5,6)=16 / 3$.

\section{Concluding remarks}

In this paper we have analyzed several functions proposed in the literature to simultaneously generalize weighted means and OWA operators. On the one hand, OWAWA operators do not generalize weighted means and OWA operators in the usual sense. On the other hand, the HWA operators are neither idempotent nor compensative while WOWA operators do not always provide the expected result. Due to the questionable behavior of these operators, we have imposed a condition to maintain the relationship among the weights and we have characterized the functions that satisfy this condition. However, the obtained functions have some problems in their definition and they are not monotonic. So, we can conclude that none of the analyzed functions is fully convincing.

\section{Acknowledgements}

I would like to thank Humberto Bustince for helpful comments and suggestions. This work is partially supported by the Spanish Ministry of Science and Innovation (Project ECO2009-07332), and ERDF.

\section{References}

1. Yager RR. On ordered weighted averaging operators in multicriteria decision making. IEEE Trans Syst, Man, Cybern 1988;18:183-190.

2. Torra V. The weighted OWA operator. Int J Intell Syst 1997;12:153-166.

3. Torra V, Narukawa Y. Modeling Decisions: Information Fusion and Aggregation Operators. Berlin: Springer, 2007. 
4. Xu ZS, Da QL. An overview of operators for aggregating information. Int J Intell Syst 2003;18:953-969.

5. Merigó JM. On the use of the OWA operator in the weighted average and its application in decision making. In: Proc World Congress on Engineering (WCE 2009); 2009. pp 82-87.

6. Merigó JM. A unified model between the weighted average and the induced OWA operator. Expert Syst Appl 2011;38:11560-11572.

7. Yager RR. Including importances in OWA aggregation using fuzzy systems modelling. IEEE Trans Fuzzy Syst 1998;6:286-294.

8. Engemann KJ, Filev DP, Yager RR. Modelling decision making using immediate probabilities. Int J Gen Syst 1996;24:281-294.

9. Xu ZS. An overview of methods for determining OWA weights. Int J Intell Syst 2005;20:843-865.

10. Fullér R. On obtaining OWA operator weights: a short survey of recent developments. In: Proc 5th IEEE Int Conf on Computational Cybernetics (ICCC 2007); 2007. pp 241-244.

11. Yager RR. Families of OWA operators. Fuzzy Sets Syst 1993;59:125-148.

12. Torra V, Lv Z. On the WOWA operator and its interpolation function. Int J Intell Syst 2009;24:1039-1056.

13. Torra V, Godo L. Averaging continuous distributions with the WOWA operator. In: Proc 2nd European Workshop on Fuzzy Decision Analysis and Neural Networks for Management, Planning and Optimization (EFDAN'97); 1997. pp 10-19.

14. Mesiar R, Novák V. Open problems from the 2nd International Conference on Fuzzy Sets Theory and its Applications. Fuzzy Sets Syst 1996;81:185-190.

15. Durante F, Sarkoci P. A note on the convex combinations of triangular norms. Fuzzy Sets Syst 2008;159:77-80.

16. Fodor JC, Rudas IJ. On continuous triangular norms that are migrative. Fuzzy Sets Syst 2007;158:1692-1697.

17. Bustince H, Montero J, Mesiar R. Migrativity of aggregation functions. Fuzzy Sets Syst 2009;160:766-777.

18. Yager RR, Filev DP. Induced ordered weighted averaging operators. IEEE Trans Syst, Man, Cybern B 1999;29:141-150. 
19. Marques Pereira R, Pasi G. On non-monotonic aggregation: mixture operators. In: Proc 4th Meeting of the EURO Working Group on Fuzzy Sets (EUROFUSE'99) and 2nd Int Conf on Soft and Intelligent Computing (SIC'99); 1999. pp 513-517.

20. Bajraktarević M. Sur une équation fonctionnelle aux valeurs moyennes. Glasnik Mat-Fiz Astronom Društvo Mat Fiz Hrvatske Ser II 1958;13:243-248.

21. Liu X. Preference solutions of probability decision making with RIM quantifiers. Int J Intell Syst 2005;20:1253-1271. 